\title{
THE UNIVERSAL KUMMER CONGRUENCES
}

\author{
SHAOFANG HONG ${ }^{\otimes}$, JIANRONG ZHAO and WEI ZHAO
}

(Received 28 June 2010; accepted 29 June 2012; first published online 22 March 2013)

Communicated by I. E. Shparlinski

Dedicated to Professor Qi Sun on the occasion of his 75th Birthday

\begin{abstract}
Let $p$ be a prime. In this paper, we present a detailed $p$-adic analysis on factorials and double factorials and their congruences. We give good bounds for the $p$-adic sizes of the coefficients of the divided universal Bernoulli number $B_{n} / n$ when $n$ is divisible by $p-1$. Using these, we then establish the universal Kummer congruences modulo powers of a prime $p$ for the divided universal Bernoulli numbers $B_{n} / n$ when $n$ is divisible by $p-1$.
\end{abstract}

2010 Mathematics subject classification: primary 11D79; secondary 11B68, 11A07.

Keywords and phrases: universal Bernoulli number, universal Kummer congruence, double factorial, $p$-adic valuation, reduced partition.

\section{Introduction}

Bernoulli numbers occur in many parts of number theory. Let $n \geq 0$ be an integer. Then the $n$th Bernoulli number is defined by the following formula:

$$
\frac{t}{e^{t}-1}=\sum_{n=0}^{\infty} \frac{B_{n}}{n !} t^{n} .
$$

From (1.1) one can see that $B_{1}=-1 / 2$ and $B_{n}=0$ for all odd $n>1$. The first few values for even $n$ are: $B_{0}=1, B_{2}=1 / 6, B_{4}=-1 / 30, B_{6}=1 / 42$, etc. The periodic behavior of the divided Bernoulli numbers $B_{n} / n$ is closely related to the existence of a $p$-adic zeta function [17]. The classical Kummer congruences [14] concern the congruence relations among the divided Bernoulli numbers $B_{n} / n$. In fact, they state that if $p$ is a prime and $(p-1) \nmid n$ and $n \equiv m \bmod p-1$, then $B_{n} / n \equiv B_{m} / m \bmod p$. One can

The first author was supported in part by the National Science Foundation of China Grant no. 10971145 and by the PhD Programs Foundation of Ministry of Education of China Grant no. 20100181110073

(C) 2013 Australian Mathematical Publishing Association Inc. 1446-7887/2013 \$16.00 
prove this congruence by means of $p$-adic measures and $p$-adic integration $[21,22]$. In [4], Baker et al. established some global-local Kummer congruences. There are also many other elegant and useful congruences, such as Wilson's theorem [2, 15], Fermat's little theorem [13, 15], Wolstenholme's theorem [23], Lucas' congruence [19] and Glaisher's congruence [8-10]. In this paper, we will mainly be concerned with the universal Kummer congruences.

In 1989, Clarke [5] introduced the concept of universal Bernoulli numbers. Assume that $c_{1}, c_{2}, \ldots$ are indeterminates over $\mathbf{Q}$. Then let $F(t)=t+c_{1} t^{2} / 2+c_{2} t^{3} / 3+\cdots$ and let $G(t)=F^{-1}(t)$ be the compositional formal power series inverse of $F(t)$, namely $F(G(t))=G(F(t))=t$. The universal Bernoulli numbers $\hat{B}_{n}$ are defined by

$$
\frac{t}{G(t)}=\sum_{n=0}^{\infty} \frac{\hat{B}_{n}}{n !} t^{n} .
$$

Obviously we have $\hat{B}_{n} \in \mathbf{Q}\left[c_{1}, c_{2}, \ldots, c_{n}\right]$. Actually $\hat{B}_{n}$ is a nontrivial Q-linear combination of all the monomials of weight $n$, where $c_{i}$ has weight $i$. So $\hat{B}_{n}$ is the sum of $p(n)$ monomials, where $p(n)$ is the partition function. Recently, Tempesta [20] introduced the universal higher-order Bernoulli polynomials and universal Bernoulli $\chi$-numbers.

Substituting $c_{i}=(-1)^{i}$, we get $F(t)=\log (1+t)$ so that $G(t)=e^{t}-1$ and we obtain the classical Bernoulli numbers $B_{n}=\hat{B}_{n}$. Miller [16] investigated the specialization where $c_{i}$ is the equivalence class of the complex projective space and proved that for this specialization, if $k$ is odd and $k \neq 1$, then $\hat{B}_{k} / k \in L$, where $L$ is the Lazard ring, a subring of $\mathbf{Q}\left[c_{1}, c_{2}, \ldots\right]$. Clarke [5] showed that the divided universal Bernoulli number $\hat{B}_{n} / n$ is $p$-integral if $(p-1) \nmid n$ which forms part of his universal von Staudt theorem. Adelberg [1] set up the universal Kummer congruences modulo a prime $p$ for the divided universal Bernoulli numbers $\hat{B}_{n} / n$ when $(p-1) \nmid n$. Soon thereafter, Adelberg [2] obtained the universal Kummer congruences modulo powers of a prime $p$ for the case $(p-1) \nmid n$. Adelberg et al. [3] established the universal Kummer congruences modulo a prime $p$ for the divided universal Bernoulli numbers when $(p-1) \mid n$. Furthermore, Adelberg et al. [3] gave a simple proof to Clarke's 1989 universal von Staudt theorem [5] which generalized the theorems of Dibag [7], Ray [18], Katz [12] and Hurwitz [11]. It is natural to ask the question of establishing the universal Kummer congruences modulo powers of a prime $p$ for the divided universal Bernoulli numbers when $(p-1) \mid n$.

In the present paper, our main goal is to investigate the above problem. We will exploit the universal Kummer congruence modulo powers of a prime $p$ for the divided universal Bernoulli numbers for the remaining case $(p-1) \mid n$. This is divided into two subcases: the even prime 2 and odd primes $p$ such that $(p-1) \mid n$. For this purpose, we need to estimate $p$-adic valuations of the coefficients of the divided universal Bernoulli number $\hat{B}_{n} / n$ when $n$ is divisible by $p-1$. The paper is organized as follows. In Section 2 we present notations and some preliminary results. In Section 3 we establish the universal Kummer congruences modulo powers of odd primes $p$ when $(p-1) \mid n$. 
Then, in Section 4, we treat the even prime $p=2$ case. We provide a detailed 2-adic analysis to many kinds of factorials and double factorials. Finally, in Section 5, we set up the universal Kummer congruences modulo powers of 2. Our result extends and strengthens the Adelberg-Hong-Ren theorem. It also extends Clarke's theorem and complements Adelberg's modulo prime powers result.

\section{Preliminaries}

If $u=\left(u_{1}, u_{2}, \ldots\right) \in \mathbf{N}^{\infty}$ with $u_{i}=0$ if $i$ is sufficiently large and $w(u):=\sum i u_{i}$, we identify $u$ with a partition of $w(u)$, where $u_{i}$ is the number of occurrences of part $i$ in the partition. If $d(u):=\sum u_{i}$, then $d(u)$ is the number of parts in the partition. We call $w(u)$ the weight of $u$ and $d(u)$ the degree of $u$. If $u_{i}=0$ for $i>n$, we write $u \in \mathbf{N}^{n}$. Let $k \geq 1$ and $l \geq 1$ be integers.

As usual, we let $v=v_{p}$ be the normalized $p$-adic valuation of $\mathbf{Q}$, that is, $v(a)=b$ if $p^{b} \mid a$ and $p^{b+1} \nmid a$. We can extend $v$ to $\mathbf{Q}\left[c_{1}, c_{2}, \ldots\right]$ by $v\left(\sum a_{u} c^{u}\right)=\min \left\{v\left(a_{u}\right)\right\}$ when $u=\left(u_{1}, \ldots, u_{n}\right) \in \mathbf{N}^{n}$ and $c_{u}=c_{1}^{u_{1}} \ldots c_{n}^{u_{n}}$. By the Lagrange inversion [5],

$$
\frac{\hat{B}_{n}}{n}=\sum_{w(u)=n} \tau_{u} c^{u},
$$

where $u=\left(u_{1}, \ldots, u_{n}\right) \in \mathbf{N}^{n}, d=d(u), c^{u}=c_{1}^{u_{1}} \ldots c_{n}^{u_{n}}$,

$$
\gamma_{u}=2^{u_{1}} \ldots(n+1)^{u_{n}} u_{1} ! \ldots u_{n} \text { ! }
$$

and

$$
\tau_{u}=(-1)^{d-1} \frac{(n+d-2) !}{\gamma_{u}} .
$$

For any positive odd integer $a$, we define the double factorial $a$ !! of $a$ by $a ! !=$ $\prod_{1 \leq k \leq a,(2, k)=1} k$. That is, $a ! !=a \cdot(a-2) \cdot \ldots \cdot 3 \cdot 1$. For a real number $x$, define $\lceil x\rceil$ to be the least integer greater than $x$ and $\lfloor x\rfloor$ to be the greatest integer less than $x$. Then $\lceil x\rceil+\lceil y\rceil \geq\lceil x+y\rceil,\lceil n+x\rceil=n+\lceil x\rceil,\lfloor x\rfloor+\lfloor y\rfloor \leq\lfloor x+y\rfloor$ and $\lfloor n+x\rfloor=n+\lfloor x\rfloor$ for any real numbers $x$ and $y$ and any integer $n$.

We will freely use the standard results listed in the following Lemma 2.1.

Lemma 2.1. We have

$$
\begin{aligned}
& v((a b) !) \geq v(a !)+v(b !), \\
& v((l p) !)=l+v(l !) .
\end{aligned}
$$

Moreover,

$$
\begin{aligned}
& v\left(\left(l p^{t}\right) !\right)=l\left(p^{t}-1\right) /(p-1)+v(l !), \\
& v(a !)=v((\lfloor a / p\rfloor p) !)=(a-s(a)) /(p-1),
\end{aligned}
$$

where $s(a):=\sum_{i=0}^{t} a_{i}$ is the base $p$ digit sum if $a=\sum_{i=0}^{t} a_{i} p^{i}$ with digits $0 \leq a_{i} \leq p-1$, and

$$
v(a !) \leq(a-1) /(p-1) \quad \text { if } a>0 .
$$


Lemma 2.2 [2]. If $p$ is an odd prime and $N=v(l)$, then

$$
(l p) ! /\left(l ! p^{l}\right) \equiv(-1)^{l} \quad \bmod p^{N+1} .
$$

Lemma 2.3 [2]. We have $v\left(\left(\sum h_{j} p^{j}\right) !\right) \geq \sum\left(j h_{j}+v\left(h_{j} !\right)\right)$.

Lemma 2.4 [2]. If $p$ is an odd prime and $0<k \leq p$, then

$$
v(a !) \geq v(a+k)
$$

unless $a=p-k$, in which case $v(a !)=v(a+k)-1$.

Clarke [5] proved some congruences about factorials. Clarke and Jones [6] obtained some stronger congruences about factorials while Adelberg et al. [3] strengthened Clarke's congruence for the $p=2$ case. But for our purpose, we need the following result due to Adelberg [2].

Lemma 2.5 [2]. Let $v(l)=N$. Then:

(i) $\quad((l+q) p) ! /\left((l+q) ! p^{l+q}\right) \equiv(-1)^{l} \cdot(q p) ! /\left(q ! p^{q}\right) \bmod p^{N+1}$;

(ii) $\quad((l+q) p+a) ! /\left((l+q) ! p^{l+q}\right) \equiv(-1)^{l} \cdot(q p+a) ! /\left(q ! p^{q}\right) \bmod p^{N+1}$;

(iii) if $a \geq e p$, then the congruence (ii) holds $\bmod p^{N+e}$;

(iv) if $a \geq(e+1) p$, then

$$
((l+q) p+a) ! /\left((l+q) ! p^{l+q+e}\right) \equiv(-1)^{l} \cdot(q p+a) ! /\left(q ! p^{q+e}\right) \bmod p^{N+1} .
$$

\section{Universal Kummer congruences modulo odd prime powers}

In the present section, we treat the odd prime case. We set up the universal Kummer congruences modulo odd prime powers. We begin with the following concept. Note that it is different from [2, Definition 4.1].

Defintion 3.1. A partition $u$ is called reduced if there is at most one part $g \in \mathbf{N}$ such that $g \neq p^{\alpha}-1, u_{g}=1$ and if $i \neq g$ and $u_{i} \neq 0$, then $i=p^{\alpha}-1$.

Lemma 3.2. Assume that $n=(m+i) p-i=i(p-1)+m p$ where $i \geq 0$. Let $w(u)=n$ and suppose that $d(u) \leq i+1$. Then there exists a reduced partition $u^{\prime}$ such that $w\left(u^{\prime}\right)=n, d\left(u^{\prime}\right) \leq i+1$ and $v\left(\tau_{u}\right) \geq v\left(\tau_{u^{\prime}}\right)$.

Proof. We first define a partition $u^{\prime \prime}:$ if $t \neq p^{\alpha}-1$, then $u_{t}^{\prime \prime}:=0$; if $t=p^{\alpha}-1$, then

$$
u_{t}^{\prime \prime}=u_{p^{\alpha}-1}^{\prime \prime}:=\sum_{\varepsilon \in \mathbf{Z}^{+}, p \nmid \varepsilon} u_{\varepsilon p^{\alpha}-1}+\delta(\alpha) \sum_{u_{j} \geq p, p \nmid(j+1)}\left(\left\lceil\frac{u_{j}}{p-1}\right\rceil-1\right),
$$

where $\delta(\alpha)=1$ for $\alpha=1$ and $\delta(\alpha)=0$ for $\alpha \geq 2$. In fact, we construct $u^{\prime \prime}$ as follows.

(i) If $u_{t} \neq 0$ with $t=\varepsilon p^{\alpha}-1$ and $p \nmid \varepsilon$ and $\varepsilon>1$, let $u_{t}^{\prime \prime}=0$ and $u_{k}^{\prime \prime}=u_{k}+u_{t}$ where $k=p^{\alpha}-1$, that is, transfer $u_{t}$ to part $p^{\alpha}-1$.

(ii) If $u_{t} \geq p$, where $p \nmid(t+1)$, let $u_{t}^{\prime \prime}=0$ and $u_{p-1}^{\prime \prime}=u_{p-1}+\left\lceil u_{t} /(p-1)\right\rceil-1$, that is, transfer to part $p-1$. 
(iii) If $0<u_{t}<p$ and $p \nmid(t+1)$, let $u_{t}^{\prime \prime}=0$, that is, delete part $t$ from the partition. The partition $u_{t}^{\prime \prime}$ is formed by considering all parts, and should be thought of as loading the parts where $i=p^{\alpha}-1$. All other parts of $u$ where (i)-(iii) do not apply are unchanged. The partition $u_{t}^{\prime \prime}$ can be constructed from $u$ one part at a time or all at once.

Clearly $w\left(u^{\prime \prime}\right) \leq n$, and if $u_{i} \neq 0$ then $i=p^{\alpha}-1$. Observe that $v\left(\gamma_{u}^{\prime \prime}\right) \geq v\left(\gamma_{u}\right)$ by (2.3) and (2.5), and that $d\left(u^{\prime \prime}\right)=d(u)$ if all modifications are of type (i), and otherwise $d\left(u^{\prime \prime}\right)<d(u)$.

Next let $g=n-w\left(u^{\prime \prime}\right) \geq 0$. If $g=0$, let $u^{\prime}=u^{\prime \prime}$. Then $u^{\prime}$ is just what we need.

If $g>0$, let $u_{g}^{\prime}=u_{g}^{\prime \prime}+1$ and $u_{j}^{\prime}=u_{j}^{\prime \prime}$ if $j \neq g$. Then $u^{\prime}$ is reduced, $w\left(u^{\prime}\right)=n, d^{\prime}=$ $d\left(u^{\prime}\right)=d\left(u^{\prime \prime}\right)+1$ and $v\left(\gamma_{u^{\prime}}\right) \geq v\left(\gamma_{u^{\prime \prime}}\right) \geq v\left(\gamma_{u}\right)$. If $d\left(u^{\prime \prime}\right)<d(u)$, then $d\left(u^{\prime}\right) \leq d(u) \leq i+1$. Hence $v\left(\tau_{u}\right) \geq v\left(\tau_{u^{\prime}}\right)$. Finally, assume that $d\left(u^{\prime \prime}\right)=d(u)$. In this case all modifications are of type (i), so

$$
n=\sum i u_{i}=\sum\left(\varepsilon p^{\alpha}-1\right) u_{\varepsilon p^{\alpha}-1}
$$

and $d=\sum u_{\varepsilon p^{\alpha}-1}$. But $n=(m+i) p-i$, thus $i \equiv \sum u_{\varepsilon p^{\alpha}-1}=d(u) \bmod p$. Since $1 \leq$ $d(u) \leq i+1$, it follows that $d(u) \leq i$ and $d\left(u^{\prime}\right)=d(u)+1 \leq i+1$. Also $n+d-2=$ $\sum \varepsilon p^{\alpha} u_{\varepsilon p^{\alpha}-1}-2$. Hence $v((n+d-2) !)=v\left(\left(n+d^{\prime}-2\right) !\right)$ by (2.4), which means that $v\left(\tau_{u}\right) \geq v\left(\tau_{u^{\prime}}\right)$. The proof is complete.

Lemma 3.3. Let $n=(m+i) p-i$ with $m=s(p-1)$. Let $u$ be reduced, with $w(u)=n$ and $d \leq i+1$. Then $v\left(\tau_{u}\right) \geq s(p-2)-1$.

Proof. Let $h_{j}=u_{p^{j}-1}$ with $j \geq 1$. Consider the following cases:

Case 1. If $u_{t} \neq 0$, then $t=p^{\alpha}-1$. In this case, $d=\sum_{j \geq 1} h_{j}$ and

$$
n=\sum_{j \geq 1} h_{j}\left(p^{j}-1\right)=(m+i) p-i .
$$

Then $i \equiv \sum_{j \geq 1} h_{j}=d \bmod p$. Since $d \leq i+1$, we may let $i=d+k p$ with $k \geq 0$. Then

$$
n+d-2=\sum_{j \geq 1} h_{j} p^{j}-2=(m+i) p-i+d-2=(m+i-k) p-2 .
$$

This implies that $k=m+i-\sum_{j \geq 1} h_{j} p^{j-1}$. It is easy to see that $v\left(\gamma_{u}\right)=\sum_{j \geq 1}\left(j h_{j}+\right.$ $\left.v\left(h_{j} !\right)\right)$. Hence by Lemma 2.3 ,

$$
\begin{aligned}
v\left(\tau_{u}\right) & =v(((m+i-k) p-2) !)-\sum_{j \geq 1}\left(j h_{j}+v\left(h_{j} !\right)\right) \\
& =m+i-k-1+v((m+i-k-1) !)-\sum_{j \geq 1}\left(j h_{j}+v\left(h_{j} !\right)\right) \\
& =m+i-k-1+v\left(\left(\sum_{j \geq 1} h_{j} p^{j-1}-1\right) !\right)-\sum_{j \geq 1}\left((j-1) h_{j}+v\left(h_{j} !\right)\right)-d \\
& =m-1+k(p-1)+\beta,
\end{aligned}
$$


where

$$
\beta=v\left(\left(\sum_{j \geq 1} h_{j} p^{j-1}-1\right) !\right)-\sum_{j \geq 1}\left((j-1) h_{j}+v\left(h_{j} !\right)\right) .
$$

For $j \geq 3$ and $h_{j} \neq 0$,

$$
\begin{aligned}
v\left(\left(h_{j} p^{j-1}-1\right) !\right) & =h_{j} p^{j-2}-1+v\left(\left(h_{j} p^{j-2}-1\right) !\right) \\
& \geq h_{j} p^{j-2}-1+v\left(\left(h_{j} p-1\right) !\right) \\
& \geq(j-1) h_{j}+v\left(h_{j} !\right)
\end{aligned}
$$

since $p^{j-2}-j+1 \geq 1$. So if there exists a $j \geq 3$ such that $h_{j} \neq 0$, then

$$
\begin{aligned}
v\left(\left(\sum_{j \geq 1} h_{j} p^{j-1}-1\right) !\right) & \geq v\left(h_{1} !\right)+v\left(\left(h_{2} p\right) !\right)+\sum_{j \geq 3} v\left(\left(h_{j} p^{j-1}-1\right) !\right) \\
& \geq \sum\left((j-1) h_{j}+v\left(h_{j} !\right)\right) .
\end{aligned}
$$

That is, $\beta \geq 0$. Hence $v\left(\tau_{u}\right) \geq m-1 \geq s(p-2)-1$ by (3.1).

If for all $j \geq 3, h_{j}=0$, since $m=s(p-1)$,

$$
n=h_{1}(p-1)+h_{2}\left(p^{2}-1\right)=m p+i(p-1)=s(p-1) p+i(p-1) .
$$

This implies that $h_{1}+h_{2}+h_{2} p=s p+i$. Since $d=h_{1}+h_{2}=i-k p$,

$$
h_{2} p=s p+\left(i-h_{1}-h_{2}\right)=s p+k p .
$$

Then $h_{2}=s+k \geq s$. On the other hand,

$$
\begin{aligned}
v\left(\tau_{u}\right) & =v\left(\left(\left(h_{1} p+h_{2} p^{2}\right)-2\right) !\right)-\left(h_{1}+2 h_{2}+v\left(h_{1} !\right)+v\left(h_{2} !\right)\right) \\
& =h_{1}+h_{2} p-1+v\left(\left(h_{1}+h_{2} p-1\right) !\right)-\left(h_{1}+2 h_{2}+v\left(h_{1} !\right)+v\left(h_{2} !\right)\right) \\
& \geq h_{2}(p-2)-1+v\left(\left(h_{2} p-1\right) !\right)-v\left(h_{2} !\right) \\
& \geq s(p-2)-1
\end{aligned}
$$

as desired.

Case 2. If there is exactly one part $g \in \mathbf{Z}^{+}$such that $g \neq p^{\alpha}-1, u_{g}=1$, and if $t \neq g$ and $u_{t} \neq 0$ then $t=p^{\alpha}-1$. In this case, we have $d=\sum_{j \geq 1} h_{j}+1$,

$$
n=\sum_{j \geq 1} h_{j}\left(p^{j}-1\right)+g=(m+i) p-i,
$$

and

$$
n+d-2=\sum_{j \geq 1} h_{j} p^{j}+g-1=(m+i) p-i+d-2 .
$$

Then $g=(m+i) p-i+d-1-\sum_{j \geq 1} h_{j} p^{j}$. 
Also we can check that

$$
v\left(\gamma_{u}\right)=\sum_{j \geq 1}\left(j h_{j}+v\left(h_{j} !\right)\right)+v(g+1) .
$$

Let $\delta=i+1-d=i-\sum_{j \geq 1} h_{j}$. Then $\delta \geq 0$ since $d \leq i+1$. We have

$$
\delta+g=(i+1-d)+(m+i) p-i+d-1-\sum_{j \geq 1} h_{j} p^{j}=k^{\prime} p
$$

where $k^{\prime}=m+i-\sum h_{j} p^{j-1}$. Then

$$
\sum_{j \geq 1} h_{j} p^{j-1}=m+i-k^{\prime}
$$

Since $g>0$ and $\delta \geq 0$, we have $k^{\prime}>0$. Furthermore, if $\delta=0$, then $g=k^{\prime} p$.

On the other hand, since $n=(m+i) p-i$,

$$
n+d-2=(m+i) p-(\delta+1)=\left(m+i-k^{\prime}\right) p+g-1 .
$$

Hence by (2.2), (2.3), (3.2), (3.3) and Lemma 2.3,

$$
\begin{aligned}
v\left(\tau_{u}\right)= & v\left(\left(\left(m+i-k^{\prime}\right) p+g-1\right) !\right)-\left(\sum_{j \geq 1}\left(j h_{j}+v\left(h_{j} !\right)\right)+v(g+1)\right) \\
\geq & v\left(\left(\left(m+i-k^{\prime}\right) p\right) !\right)+v((g-1) !) \\
& \quad-\sum_{j \geq 1}\left((j-1) h_{j}+v\left(h_{j} !\right)\right)-\sum_{j \geq 1} h_{j}-v(g+1) \\
\geq & m+i-k^{\prime}-\sum_{j \geq 1} h_{j}+v\left(\left(m+i-k^{\prime}\right) !\right)+v((g-1) !) \\
& \quad-v\left(\left(\sum_{j \geq 1} h_{j} p^{j-1}\right) !\right)-v(g+1) \\
\geq & m+i-k^{\prime}-\sum_{j \geq 1} h_{j}+v((g-1) !)-v(g+1) \\
\geq & m-1+\delta^{\prime} \\
\geq & s(p-2)-1+\delta^{\prime},
\end{aligned}
$$

where

$$
\delta^{\prime}=\delta-k^{\prime}+1+v((g-1) !)-v(g+1) .
$$

Thus it is sufficient to show that $\delta^{\prime} \geq 0$.

If $\delta-k^{\prime} \geq 0$, then by Lemma 2.4,

$$
\delta^{\prime} \geq v((g-1) !)-v(g+1)+1 \geq 0 .
$$


It remains to consider the case $\delta-k^{\prime}<0$. In this case, let $\delta=x p+r$ with $0 \leq r<p$. Then $x=\lfloor\delta / p\rfloor$. Clearly $x<\delta$ unless $\delta=0=r=x$, and $x<\delta-1$ if $\delta \geq 2$ since $p \geq 3$.

Since $0<g=k^{\prime} p-\delta=\left(k^{\prime}-x\right) p-r$, we have $k^{\prime}-x \geq 1$. But

$$
g-1=\left(k^{\prime}-x\right) p-(r+1)=\left(k^{\prime}-x-1\right) p+(p-r-1) .
$$

So

$$
v((g-1) !)=v\left(\left(\left(k^{\prime}-x-1\right) p\right) !\right)=k^{\prime}-x-1+v\left(\left(k^{\prime}-x-1\right) !\right) .
$$

Hence

$$
\begin{aligned}
\delta^{\prime} & =\delta-k^{\prime}+1+k^{\prime}-x-1+v\left(\left(k^{\prime}-x-1\right) !\right)-v(g+1) \\
& =\delta-x+v\left(\left(k^{\prime}-x-1\right) !\right)-v(g+1) .
\end{aligned}
$$

If $p \nmid g+1$, then $\Delta^{\prime}=\delta-x \geq 0$. Now let $p \mid g+1$. Since $g+1=\left(k^{\prime}-x\right) p-(r-1)$, we have $r=1$ and $g+1=\left(k^{\prime}-x\right) p$. So

$$
\delta^{\prime}=\delta-x+v\left(\left(k^{\prime}-x-1\right) !\right)-v\left(k^{\prime}-x\right)-1 .
$$

Since $r \neq 0$, we have $\delta \neq 0$. So $\delta-x \geq 1$. By Lemma 2.4 it remains to show that $\delta-x=1$ is impossible. If $\delta-x=1$, then $\delta=1$ and $x=0$, and it is sufficient to show that $k^{\prime}-x \neq p$. But if $k^{\prime}=p$ then $\delta+g=1+g=k^{\prime} p=p^{2}$, so $g=p^{2}-1$, which is impossible since $g \neq p^{\alpha}-1$. The proof of Lemma 3.3 is complete.

Corollary 3.4. Let $n=(m+i) p-i$ with $m=s(p-1)$. Assume that $w(u)=n$ and $d(u) \leq i+1$. Then $v\left(\tau_{u}\right) \geq s(p-2)-1$.

PRoof. This corollary follows immediately from Lemmas 3.2 and 3.3.

Let us now recall the critical bounds for $\hat{B}_{n} / n$.

Lemma 3.5 [3]. Let $p$ be an odd prime. Suppose that $w(u)=n, n=(p-1) s_{0}$ and $u_{p-1}<s_{0}$. Let $e=v\left(\gamma_{u}\right)-v\left(\left(p u_{p-1}\right) !\right)$ and $\dot{n}=n-(p-1) u_{p-1}$. Then

$$
n+d-2 \geq\left(u_{p-1}+e+1\right) p
$$

except for the following cases where

$$
\left(u_{p-1}+e+1\right) p>n+d-2 \geq\left(u_{p-1}+e\right) p:
$$

(i) $\quad p \geq 3, u_{p-1}=s_{0}-1$, and for $1 \leq i \leq(p-3) / 2$, we have $u_{i}=u_{p-1-i}$, or if $i=$ $(p-1) / 2$, we have $u_{i}=2$. In these cases, $\dot{n}=p-1$ and $e=0$.

(ii) $\quad p=3, u_{2}=s_{0}-4, u_{8}=1$. In this case, $\dot{n}=8$ and $e=2$.

For any positive integer $x$, by Lemma 2.2,

$$
\frac{(x p) !}{x ! p^{x}}-(-1)^{x} \equiv 0 \quad \bmod p^{v(x)+1} .
$$


Then associated to the prime number $p$, we can define an arithmetical function $k_{p}$ for any positive integer $x$ by

$$
k_{p}(x):=\frac{1}{p^{v(x)+1}}\left(\frac{(x p) !}{x ! p^{x}}-(-1)^{x}\right) .
$$

Clearly $k_{p}(x) \in \mathbf{Z}$ for any positive integer $x$ and $k_{p}(1)$ is the usual Wilson quotient (see, for instance, [15]). We call $k_{p}(x)$ the generalized Wilson quotient. On the other hand, for any positive integer $x$, we define

$$
h_{p}(x):=\frac{(-1)^{x-1}(x p-2) !}{p^{x} x !} .
$$

Then

$$
h_{p}(x)=\frac{(-1)^{x-1}}{p x} \prod_{i=1, p \nmid i}^{x p-2} i .
$$

We have the following result.

Lemma 3.6. Let $s$ and $l$ be positive integers such that $v_{p}(l)=N$. If $v(s)<N$, then

$$
h_{p}(l+s)-h_{p}(s) \equiv g_{p}(l, s) \quad \bmod p^{N+1},
$$

where $g_{p}(l, s)$ is defined by

$$
g_{p}(l, s):=\left((-1)^{l} s k_{p}(l) p^{N+1}-(-1)^{s} l k_{p}(s) p^{v(s)+1}-l\right) /(p s(l+s)) \quad \bmod p^{N+1} .
$$

Proof. First since $v(s)<N$,

$$
\begin{aligned}
\prod_{i=1, p \nmid i}^{(l+s) p-2} i & =\left(\prod_{i=1, p \nmid i}^{l p-1} i\right)\left(\prod_{i=l p+1, p \nmid i}^{(l+s) p-2} i\right) \\
& =\left(\prod_{i=1, p \nmid i}^{l p-1} i\right)\left(\prod_{i=1, p \nmid i}^{s p-2}(l p+i)\right) \\
& =\left(\prod_{i=1, p \nmid i}^{l p-1} i\right)\left(\prod_{i=1, p \nmid i}^{s p-2} i+l p\left(\prod_{i=1, p \nmid i}^{s p-2} i\right)\left(\sum_{j=1, p \nmid j}^{s p-2} j^{-1}\right)+(l p)^{2} k_{1}\right) \\
& =\left(\prod_{i=1, p \nmid i}^{l p-1} i\right)\left(\left(\prod_{i=1, p \nmid i}^{s p-2} i\right)\left(1+l p+(l p / 2) \sum_{j=2, p \nmid j}^{s p-2}\left(j^{-1}+(s p-j)^{-1}\right)\right)+(l p)^{2} k_{1}\right) \\
& =\left(\prod_{i=1, p \nmid i}^{l p-1} i\right)\left(\left(\prod_{i=1, p \nmid i}^{s p-2} i\right)\left(1+l p+l p(s p / 2) \sum_{j=2, p \nmid j}^{s p-2}(j(s p-j))^{-1}\right)+(l p)^{2} k_{1}\right) \\
& \equiv(1+l p)\left(\prod_{i=1, p \nmid i}^{l p-1} i\right)\left(\prod_{i=1, p \nmid i}^{s p-2} i\right) \bmod p^{v(s)+N+2},
\end{aligned}
$$

where $k_{1}$ is some positive integer. 
Consequently, by the definition of $k_{p}(s)$ we can easily show that

$$
\prod_{i=1, p \nmid i}^{l p-1} i=\frac{(l p) !}{l ! p^{l}}=(-1)^{l}+k_{p}(l) p^{N+1}
$$

and

$$
\prod_{i=1, p \nmid i}^{s p-2} i=(-1)(1-s p)^{-1}(s p) ! /\left(s ! p^{s}\right)=\left(-\sum_{j=0}^{\infty}(s p)^{j}\right)\left((-1)^{s}+k_{p}(s) p^{v(s)+1}\right) .
$$

Then by (3.4), (3.5) and (3.6) we can deduce that

$$
\begin{aligned}
& h_{p}(l+s)-h_{p}(s) \\
& =(-1)^{l+s-1}(p(l+s))^{-1} \prod_{i=1, p \nmid i}^{(l+s) p-2} i-(-1)^{s-1}(p s)^{-1} \prod_{i=1, p \nmid i}^{s p-2} i \\
& \equiv(-1)^{l+s-1}(p(l+s))^{-1}(1+l p)\left(\prod_{i=1, p \nmid i}^{l p-1} i\right)\left(\prod_{i=1, p \nmid i}^{s p-2} i\right)-(-1)^{s-1}(p s)^{-1} \prod_{i=1, p \nmid i}^{s p-2} i \\
& =(-1)^{s-1}(p s)^{-1}\left(\prod_{i=1, p \nmid i}^{s p-2} i\right)\left((-1)^{l} s(1+l p)(l+s)^{-1} \prod_{i=1, p \nmid i}^{l p-1} i-1\right) \\
& =(-1)^{s-1}(p s)^{-1}\left(\prod_{i=1, p \nmid i}^{s p-2} i\right)\left((-1)^{l} s(1+l p)(l+s)^{-1}\left((-1)^{l}+k_{p}(l) p^{N+1}\right)-1\right) \\
& \equiv(-1)^{s-1}(p s)^{-1}\left(\prod_{i=1, p \nmid i}^{s p-2} i\right)\left(s(l+s)^{-1}\left((-1)^{l} k_{p}(l) p^{N+1}+l p\right)-l(l+s)^{-1}\right) \\
& \equiv(-1)^{s-1}\left(\prod_{i=1, p \nmid i}^{s p-2} i\right)\left((-1)^{l} k_{p}(l) p^{N}(l+s)^{-1}+l(l+s)^{-1}-l(p s(l+s))^{-1}\right) \\
& =(-1)^{s}\left(\sum_{j=0}^{\infty}(s p)^{j}\right)\left((-1)^{s}+k_{p}(s) p^{v(s)+1}\right)\left(\left((-1)^{l} k_{p}(l) p^{N}+l\right)(l+s)^{-1}-l(p s(l+s))^{-1}\right) \\
& \equiv(1+s p)\left(1+(-1)^{s} k_{p}(s) p^{v(s)+1}\right)\left(\left((-1)^{l} k_{p}(l) p^{N}+l\right)(l+s)^{-1}-l(p s(l+s))^{-1}\right) \\
& \equiv g_{p}(l, s) \bmod p^{N+1}
\end{aligned}
$$

as required. Thus Lemma 3.6 is proved.

Using the generalized Wilson quotient, we can now establish the universal Kummer congruences modulo powers of odd primes. This is the first main result of this paper.

Theorem 3.7. Let $n=m+l(p-1)$ and $p^{N} \mid l$. Suppose that $m=s(p-1)$ and $s \geq$ $\lceil(N+2) /(p-2)\rceil$. 
(i) If $p \geq 5$, then

$$
\hat{B}_{n} / n \equiv c_{p-1}^{l} \hat{B}_{m} / m+g_{p}(l, s) c_{p-1}^{l+s} \quad \bmod p^{N+1} \mathbf{Z}_{p}\left[c_{1}, \ldots, c_{n}\right] .
$$

(ii) If $p=3$, then

$$
\hat{B}_{n} / n \equiv c_{2}^{l} \hat{B}_{m} / m+g_{3}(l, s) c_{2}^{l+s}+\Psi c_{2}^{l+s-4} c_{8} \quad \bmod 3^{N+1} \mathbf{Z}_{3}\left[c_{1}, \ldots, c_{n}\right],
$$

where $\Psi=0$ if $s \equiv 1 \bmod 3, \Psi=-l$ if $s \equiv 0 \bmod 3$, and $\Psi=l$ if $s \equiv-1 \bmod 3$.

Proof. First consider the terms $\tau_{u} c^{u}$ of $\hat{B}_{n} / n$ where $u_{p-1} \geq l$. Let $u_{p-1}^{\prime}=u_{p-1}-l:=q$ and $u_{i}^{\prime}=u_{i}$ for $i \neq p-1$. Then $w\left(u^{\prime}\right)=m$ and $c^{u^{\prime}} c_{p-1}^{l}=c^{u}$. It follows from (2.1) that

$$
\frac{\hat{B}_{n}}{n}-c_{p-1}^{l} \frac{\hat{B}_{m}}{m}=\sum_{w(u)=n, u_{p-1} \geq l}\left(\tau_{u}-\tau_{u^{\prime}}\right) c^{u}+\sum_{w(u)=n, u_{p-1}<l} \tau_{u} c^{u} .
$$

If $u_{p-1}=n /(p-1)=l+s$, then $u_{p-1}^{\prime}=s=m /(p-1)$. Hence by the definition and Lemma 3.6,

$$
\tau_{u}-\tau_{u^{\prime}}=h_{p}(l+s)-h_{p}(s) \equiv g_{p}(l, s) \bmod p^{N+1} .
$$

Next assume that $u_{p-1}<l+s$. Now let $\dot{u}_{p-1}=0$ and $\dot{u}_{i}=u_{i}$ for $i \neq p-1$.

Let $v\left(\gamma_{\dot{u}}\right)=e$. Then $\gamma_{\dot{u}}=\varepsilon p^{e}$ where $p \nmid \varepsilon$. Since $d^{\prime}=d\left(u^{\prime}\right)=d(u)-l$ and $\dot{d}=d(\dot{u})=$ $d^{\prime}-q$,

$$
n+d-2=l p+m+d^{\prime}-2=l p+q p+\dot{n}+\dot{d}-2 .
$$

Also $\gamma_{u}=(l+q) ! p^{l+q} \gamma_{\dot{u}}$ and $\gamma_{u^{\prime}}=q ! p^{q} \gamma_{\dot{u}}$.

If $e=0$, then $\left(\gamma_{\dot{u}}, p\right)=1$. So applying Lemma 2.5(ii) with $a=\dot{n}+\dot{d}-2$ gives us

$$
\tau_{u} \equiv \tau_{u^{\prime}} \quad \bmod p^{N+1} .
$$

If $e>0$, then by Lemma 3.5 we have $\dot{n}+\dot{d}-2 \geq(e+1) p$ with the exception of case (ii) where $\dot{n}=8, e=2$, and $u_{2}=l+s-4, u_{8}=1$. Hence by Lemma 2.5(iv), $\tau_{u} \equiv \tau_{u^{\prime}} \bmod p^{N+1}$ without the exceptional case.

We now turn to the exceptional term, which occurs if and only if $p=3, u_{2}=$ $l+s-4, u_{8}=1$. In this case, $d=l+s-4+1=l+s-3, d^{\prime}=s-3$. Then

$$
n+d-2=2(l+s)+l+s-3-2=3(l+s)-5
$$

and

$$
n^{\prime}+d^{\prime}-2=m+d^{\prime}-2=3 s-5 \text {. }
$$

Also

$$
\gamma_{u}=3^{l+s-4} \cdot 9 \cdot(l+s-4) !=3^{l+s-2}(l+s-4) !
$$

and

$$
\gamma_{u^{\prime}}=3^{s-4} \cdot 9 \cdot(s-4) !=3^{s-2}(s-4) !
$$


Hence by Lemmas 2.2 and 2.5(ii),

$$
\begin{aligned}
\tau_{u}= & (-1)^{d-1}(3(l+s)-5) ! 3^{-(l+s-2)}((l+s-4) !)^{-1} \\
= & (-1)^{d-1}(3(l+s)-5) ! 3^{-(l+s-2)}((l+s-2) !)^{-1}(l+s-3)(l+s-2) \\
= & (-1)^{d-1}(3(l+s)-5) ! 3^{-(l+s-2)}((l+s-2) !)^{-1}\left(l^{2}+l(2 s-5)+(s-2)(s-3)\right) \\
\equiv & (-1)^{d-1}(-1)^{l}(3 s-5) ! 3^{2-s}((s-2) !)^{-1} l(2 s-5) \\
& \quad+(-1)^{d-1}(-1)^{l}(3 s-5) ! 3^{2-s}((s-2) !)^{-1}(s-2)(s-3) \\
& \equiv(-1)^{s-4} l(3 s-5) ! 3^{2-s}((s-2) !)^{-1}(2 s-5)+\tau_{u^{\prime}} \quad \bmod 3^{N+1} .
\end{aligned}
$$

But by Lemma 2.1,

$$
\begin{aligned}
(3 s-5) ! 3^{2-s}((s-2) !)^{-1}(2 s-5) & =(3 s-6) ! 3^{2-s}((s-2) !)^{-1}(3 s-5)(2 s-5) \\
& \equiv(-1)^{s-2}(-1)(2 s-2) \bmod 3 .
\end{aligned}
$$

So

$$
\tau_{u} \equiv(-1)^{s-4+s-2+1}(2 s-2) l+\tau_{u^{\prime}}=(-1) l(2 s-2)+\tau_{u^{\prime}} \bmod 3^{N+1} .
$$

Thus $\tau_{u} \equiv \tau_{u}^{\prime} \bmod 3^{N+1}$ if $s \equiv 1 \bmod 3, \tau_{u} \equiv \tau_{u}^{\prime}-l \bmod 3^{N+1}$ if $s \equiv 0 \bmod 3$ and $\tau_{u} \equiv \tau_{u^{\prime}}+l \bmod 3^{N+1}$ if $s \equiv-1 \bmod 3$.

We can now deal with the terms where $u_{p-1}<l$. In what follows we assume that $u_{p-1}=l-x$ with $x \geq 1$. To finish the proof, it is sufficient to show that if $w(u)=n$ and $u_{p-1}<l$ then $\tau_{u} \equiv 0 \bmod p^{N+1}$ if $s \geq\lceil(N+2) /(p-2)\rceil$, with the single exception where $p=3$ and $u$ is given by $u_{p-1}=l+s-4, u_{8}=1$.

If $n+d-2 \geq l p$, then there exists an integer $k$ such that

$$
(l+k) p \leq n+d-2<(l+k+1) p .
$$

With our usual notation,

$$
\dot{n}=n-(l-x)(p-1)=m+x(p-1) .
$$

Let $e=v\left(\gamma_{\dot{u}}\right)$. Since $u_{p-1}=l-x<l$,

$$
n+d-2 \geq(l-x+e+1) p
$$

with the exceptional case (ii) of Lemma 3.5 which was previously considered.

On the other hand,

$$
\begin{aligned}
v\left(\tau_{u}\right) & =v((n+d-2) !)-(l-x)-v((l-x) !)-e \\
& \geq v(((l+k) p) !)-(l-x)-v((l-x) !)-e \\
& =(l+k)+v((l+k) !)-(l-x)-v((l-x) !)-e \\
& \geq k+x+v(l)-e .
\end{aligned}
$$


Suppose now that $v\left(\tau_{u}\right)<v(l p)$. Then $e>k+x-1$, that is, $e \geq k+x$. But by (3.7),

$$
n+d-2 \geq(l-x+e+1) p \geq(l-x+k+x+1) p=(l+k+1) p
$$

which contradicts $(l+k+1) p>n+d-2$. Thus in this case we infer that $v\left(\tau_{u}\right) \geq$ $v(l p) \geq N+1$.

Now it remains to consider the case $n+d-2<l p$. In this case,

$$
n+d=m+l(p-1)+d<l p+2,
$$

that is, $m+d \leq l+1$. Since $u_{p-1}=l-x<d \leq l+1-m, x \geq m$. One may let $x=m+i$ with $i \geq 0$. Then

$$
w(\dot{u})=n-u_{p-1}(p-1)=m+x(p-1)=(m+i) p-i
$$

and

$$
d(\dot{u})=d-(l-x) \leq x+1-m=i+1 .
$$

But $u_{p-1}=l-x$. So $n+d-2 \geq(l-x) p+\dot{n}+\dot{d}-2$ and $v\left(\tau_{u}\right) \geq v\left(\tau_{\dot{u}}\right)$. Since $s \geq$ $\lceil(N+2) /(p-2)\rceil$, then by assumption and replacing $n$ and $u$ by $\dot{n}=(m+i) p-i$ and $\dot{u}$, respectively, Corollary 3.4 gives $\tau_{u} \equiv 0 \bmod p^{N+1}$.

The proof of Theorem 3.7 is complete.

\section{Auxiliary results for powers of 2}

In the current section, we deal with the even prime $p=2$ case. In order to establish the universal Kummer congruences modulo powers of 2, we provide a detailed 2adic analysis for many kinds of factorials and double factorials. First we give several congruences modulo powers of 2 for double factorials.

LEMMA 4.1 [3]. Each of the following is true.

(i) If $k$ is odd, then $(2 k-1) ! ! \equiv(-1)^{(k-1) / 2} \bmod 4$.

(ii) If $k \geq 1$, then $(4 k-3) ! ! \equiv(-1)^{k-1} \bmod 16$.

(iii) If $k \geq 1$ and $N \geq 3$, then $\left(k 2^{N}-3\right) ! ! \equiv-1 \bmod 2^{N+1}$.

Lemma 4.2. Let $k \geq 1, N \geq 3$ and $a \geq 2$ be integers. Then:

(i) $\quad\left(k 2^{N}+2 a-3\right) ! ! /\left(k 2^{N}+1\right) ! ! \equiv \begin{cases}(2 a-3) ! ! \bmod 2^{N+1+\min \{v(a), N-1\}} & \text { if } 2 \mid a, \\ (2 a-3) ! !+k 2^{N} \bmod 2^{N+1} & \text { if } 2 \nmid a ;\end{cases}$

(ii) $\quad\left(k 2^{N}+2 a-3\right) ! ! \equiv\left\{\begin{array}{lll}(2 a-3) ! ! & \bmod 2^{N+1} & \text { if } 2 \mid a, \\ (2 a-3) ! !+k 2^{N} & \bmod 2^{N+1} & \text { if } 2 \nmid a ;\end{array}\right.$

(iii) If $k$ is odd,

$$
\left(k 2^{N}-3\right) ! ! \equiv\left\{\begin{array}{lll}
-1+(-1)^{(k-1) / 2} 2^{N+1} & \bmod 2^{N+3} & \text { if } N=3, \\
-1+(-1)^{(k+1) / 2} 2^{N+1} & \bmod 2^{N+3} & \text { if } N \geq 4
\end{array}\right.
$$

In particular, we have $\left(k 2^{N}-3\right) ! ! \equiv-1+2^{N+1} \bmod 2^{N+2}$. 
Proof. (i) First

$$
\begin{aligned}
\left(k 2^{N}+2 a-3\right) ! ! /\left(k 2^{N}+1\right) ! ! & =\left(k 2^{N}+3\right)\left(k 2^{N}+5\right) \cdots\left(k 2^{N}+2 a-3\right) \\
& =(2 a-3) ! !+k 2^{N} \sum_{j \in S}(2 a-3) ! ! / j+x\left(k 2^{N}\right)^{2} \\
& \equiv(2 a-3) ! !+k 2^{N} \sum_{j \in S}(2 a-3) ! ! / j \quad \bmod 2^{2 N},
\end{aligned}
$$

where $x \in \mathbf{N}$ and $S=\{3,5, \ldots, 2 a-3\}$. If $j \in S$, then $2 a-j \in S$. Clearly $j \neq 2 a-j$ unless $j=a \in S$. If $2 \mid a$, then $a \notin S$. Therefore

$$
\begin{aligned}
\sum_{j \in S} \frac{(2 a-3) ! !}{j} & =\sum_{j \in S, j<a}\left(\frac{(2 a-3) ! !}{2 a-j}+\frac{(2 a-3) ! !}{j}\right) \\
& =2 a \sum_{j \in S, j<a} \frac{(2 a-3) ! !}{j(2 a-j)} .
\end{aligned}
$$

Then

$$
v\left(k 2^{N} \sum_{j \in S} \frac{(2 a-3) ! !}{j}\right) \geq N+1+v(a) .
$$

Hence by (4.1), Lemma 4.2(i) is true for the case $2 \mid a$.

Now we consider the case $2 \nmid a$, then $a \in S$. Hence

$$
\begin{aligned}
\sum_{j \in S} \frac{(2 a-3) ! !}{j} & =\sum_{j \in S, j<a}\left(\frac{(2 a-3) ! !}{2 a-j}+\frac{(2 a-3) ! !}{j}\right)+\frac{(2 a-3) ! !}{a} \\
& =2 a \sum_{j \in S, j<a} \frac{(2 a-3) ! !}{j(2 a-j)}+\frac{(2 a-3) ! !}{a} .
\end{aligned}
$$

Then $v\left(\sum_{j \in S}(2 a-3) ! ! / j\right)=0$ since $v((2 a-3) ! ! / a)=0$. Thus by (4.1) we infer that Lemma 4.2(i) holds for the case $2 \nmid a$. Part (i) is proved.

(ii) Since $N \geq 3,2 N \geq N+1$. Then by Lemma 4.1(iii),

$$
\begin{aligned}
\left(k 2^{N}+1\right) ! ! & =\left(k 2^{N}-3\right) ! !\left(k 2^{N}-1\right)\left(k 2^{N}+1\right) \\
& \equiv-\left(k 2^{N}-3\right) ! ! \\
& \equiv 1 \quad \bmod 2^{N+1} .
\end{aligned}
$$

So the desired result follows immediately from part (i).

(iii) If $k \equiv 1 \bmod 4$, then by part (ii),

$$
\left(k 2^{N}-3\right) ! !=\left(t_{1} 2^{N+2}+2^{N}-3\right) ! ! \equiv\left(2^{N}-3\right) ! ! \bmod 2^{N+3},
$$

where $t_{1} \geq 0$ is an integer. If $k \equiv 3 \bmod 4$, then by part (ii),

$$
\left(k 2^{N}-3\right) ! !=\left(t_{2} 2^{N+2}+3 \cdot 2^{N}-3\right) ! ! \equiv\left(3 \cdot 2^{N}-3\right) ! ! \bmod 2^{N+3},
$$


where $t_{2} \geq 0$ is an integer. By part (i),

$$
\begin{aligned}
&\left(3 \cdot 2^{N}-3\right) ! ! \equiv\left(2^{N+1}+2^{N}-3\right) ! !\left(\left(2^{N+1}+1\right) ! !\right)^{-1}(-1) \\
& \quad \times\left(2^{N}+2^{N}-3\right) ! !\left(\left(2^{N}+1\right) ! !\right)^{-1}(-1)\left(2^{N}-3\right) ! ! \\
& \equiv\left(\left(2^{N}-3\right) ! !\right)^{3} \bmod 2^{2 N} .
\end{aligned}
$$

Since $N \geq 3,2 N \geq N+3$. Then by (4.3) and (4.4),

$$
\left(k 2^{N}-3\right) ! ! \equiv\left(\left(2^{N}-3\right) ! !\right)^{3} \quad \bmod 2^{N+3} .
$$

First consider the case $N=3$. Clearly $\left(2^{3}-3\right) ! ! \equiv 15 \bmod 2^{6}$. Hence by (4.2),

$$
\left(k 2^{3}-3\right) ! ! \equiv 15=-1+(-1)^{(k-1) / 2} 2^{4} \bmod 2^{6}
$$

if $k \equiv 1 \bmod 4 . \mathrm{By}(4.5)$,

$$
\left(k \cdot 2^{3}-3\right) ! ! \equiv 15^{3} \equiv-17=-1+(-1)^{(k-1) / 2} 2^{4} \quad \bmod 2^{6}
$$

if $k \equiv 3 \bmod 4$. Part (iii) is proved for the $N=3$ case. In what follows we deal with the $N \geq 4$ case.

We claim that $\left(2^{N}-3\right) ! ! \equiv-1-2^{N+1} \bmod 2^{N+3}$ for $N \geq 4$. We use induction on $N$. Evidently $\left(2^{4}-3\right)$ !! $\equiv-1-2^{5} \bmod 2^{7}$ if $N=4$. Assume that $\left(2^{N}-3\right) ! !$ $-1-2^{N+1} \bmod 2^{N+3}$ for some $N \geq 4$. Since $N \geq 4,2 N \geq N+4$. Then by part (i) and the induction hypothesis,

$$
\begin{aligned}
\left(2^{N+1}-3\right) ! ! & \equiv\left(2^{N}+2^{N}-3\right) ! !\left(\left(2^{N}+1\right) ! !\right)^{-1}(-1)\left(2^{N}-3\right) ! ! \\
& \equiv-\left(\left(2^{N}-3\right) ! !\right)^{2} \\
& \equiv-1-2^{N+2} \bmod 2^{N+4}
\end{aligned}
$$

Hence the claim is true. So the desired result follows immediately from the claim and (4.2), (4.5). Part (iii) is proved.

\section{Lemma 4.3. Let $a \geq 0$ and $i \geq 1$ be integers. Define}

$$
f_{a}(i, j):=(a+1) \cdots(a+2 i) /(a+j)
$$

for $j \in\{1, \ldots, 2 i\}$. Then

$$
v\left(\sum_{j=1}^{2 i} f_{a}(i, j)\right) \geq \begin{cases}i-1 & \text { if } 1 \leq i \leq 3 \\ i & \text { otherwise }\end{cases}
$$

Proof. First

$$
v\left(f_{a}(1,1)+f_{a}(1,2)\right)=v(2 a+3)=0 .
$$


So Lemma 4.3 is true for $i=1$. For $i=2$, we can easily check that

$$
\sum_{j=1}^{4} f_{a}(2, j) \equiv 2 a(a+1)+2 \equiv 2 \bmod 4 .
$$

Hence $v\left(\sum_{j=1}^{4} f_{a}(2, j)\right)=1$ as required. Lemma 4.3 is true when $i=2$. For $i=3$, by the fact that 8 divides the product of any four consecutive integers, we get $8 \mid f_{a}(3, j)$ for $j=1,2,5,6$. On the other hand,

$$
f_{a}(3,3) \equiv a^{3}(a+1)^{2} \equiv 0 \quad \bmod 4
$$

and

$$
f_{a}(3,4) \equiv a^{2}(a+1)^{2}(a+3) \equiv 0 \quad \bmod 4 .
$$

It follows that

$$
v\left(\sum_{j=1}^{6} f_{a}(3, j)\right) \geq \min _{1 \leq j \leq 6}\left\{v\left(f_{a}(3, j)\right)\right\} \geq 2
$$

as required.

Now let $i \geq 4$. We may let $a=2 b+b^{\prime}$, where $b \in \mathbf{Z}_{\geq 0}$ and $b^{\prime}=0$ or 1 . Then

$$
\begin{aligned}
v\left(f_{a}(i, j)\right) & =v\left((2 b+2) \cdots(2 b+2 i) /\left(2 b+j+b^{\prime}\right)\right) \\
& =i+v\left((b+1) \cdots(b+i) /\left(2 b+j+b^{\prime}\right)\right) .
\end{aligned}
$$

If $2 \nmid\left(b^{\prime}+j\right)$, then

$$
v\left((b+1) \cdots(b+i) /\left(2 b+j+b^{\prime}\right)\right) \geq 3 .
$$

If $2 \mid\left(b^{\prime}+j\right)$, then

$$
b+\left(j+b^{\prime}\right) / 2 \in\{b+1, \ldots, b+i\}
$$

Thus

$$
v\left((b+1) \cdots(b+i)\left(2\left(b+\left(j+b^{\prime}\right) / 2\right)\right)^{-1} \geq 0\right.
$$

since $i \geq 4$. Hence by (4.6), we have $v\left(f_{a}(i, j)\right) \geq i$ for all $1 \leq j \leq 2 i$. Therefore

$$
v\left(\sum_{j=1}^{2 i} f_{a}(i, j)\right) \geq \min _{1 \leq j \leq 2 i}\left\{\left(v\left(f_{a}(i, j)\right)\right)\right\} \geq i .
$$

The proof of Lemma 4.3 is complete.

REMARK 4.4. In fact, we infer that

$$
v\left((b+1) \cdots(b+i)\left(2 b+j+b^{\prime}\right)^{-1}\right) \geq 1
$$

for $i \geq 6$ and

$$
v\left((b+1) \cdots(b+i)\left(2 b+j+b^{\prime}\right)^{-1}\right) \geq 3
$$

for $i \geq 8$. Then $v\left(f_{a}(i, j)\right) \geq i+1$ for $i \geq 6$ and $v\left(f_{a}(i, j)\right) \geq i+3$ for $i \geq 8$ by (4.6). 
Lemma 4.5. Let $q, r, a \in \mathbf{N}, e \in \mathbf{Z}^{+}$and $v(l)=N$. Define $\delta_{r}=l$ for $r=1,2$ and $\delta_{r}=0$ for $r \neq 1,2$. Each of the the following is true.

(i) $(l+q+2 r) ! /((l+q) ! r !) \equiv(q+2 r) ! /(q ! r !)+\delta_{r} \bmod 2^{N+1}$.

(ii) If $a=0$ or 1 , then

$$
\begin{aligned}
& (2(l+q+2 r)+a) ! /\left(2^{l+q+2 r}(l+q) ! r !\right) \\
& \quad \equiv(2(q+2 r)+a) ! /\left(2^{q+2 r} q ! r !\right)+\delta_{r} \quad \bmod 2^{N+1} .
\end{aligned}
$$

(iii) If $a \geq 2 e$, then

$$
(2(l+q+2 r)+a) ! /\left(2^{l+q+2 r}(l+q) ! r !\right) \equiv(2(q+2 r)+a) ! /\left(2^{q+2 r} q ! r !\right) \bmod 2^{N+e} .
$$

(iv) If $a \geq 2(e+1)$, then

$$
\begin{aligned}
& (2(l+q+2 r)+a) ! /\left(2^{l+q+2 r+e}(l+q) ! r !\right) \\
& \quad \equiv(2(q+2 r)+a) ! /\left(2^{q+2 r+e} q ! r !\right) \bmod 2^{N+1} .
\end{aligned}
$$

Proof. (i) For $r=0$, the congruence is trivial. Now let $r>0$. If $r \geq N+1$,

$$
v((l+q+2 r) ! /((l+q) ! r !)) \geq v((2 r) !)-v(r !)=r \geq N+1
$$

since

$$
v((l+q+2 r) !) \geq v((l+q) !)+v((2 r) !)
$$

and $v((2 r) !)=r+v(r !)$. Similarly, we have $v((q+2 r) ! /(q ! r !)) \geq N+1$. Then

$$
(l+q+2 r) ! /((l+q) ! r !) \equiv(q+2 r) ! /(q ! r !) \equiv 0 \quad \bmod 2^{N+1}
$$

as required. If $r \leq N$, then $v(r !) \leq r-1 \leq N-1$. On the other hand,

$$
\begin{aligned}
(l+q & +2 r) ! /((l+q) ! r !)=(l+q+1) \cdots(l+q+2 r) / r ! \\
& \equiv(q+1) \cdots(q+2 r) / r !+(l / r !) \sum_{j=1}^{2 r}(q+1) \cdots(q+2 r) /(q+j) \\
& \equiv(q+2 r) ! /(q ! r !)+(l / r !) \sum_{j=1}^{2 r} f_{q}(r, j) \bmod 2^{N+1},
\end{aligned}
$$

where $f_{a}(i, j)$ is defined as in Lemma 4.3. From (4.7) and Lemma 4.3 we deduce that if $r=1,2$, then

$$
(l+q+2 r) ! /((l+q) ! r !) \equiv(q+2 r) ! /(q ! r !)+l \quad \bmod 2^{N+1},
$$

and if $r \geq 3$, then

$$
(l+q+2 r) ! /((l+q) ! r !) \equiv(q+2 r) ! /(q ! r !) \bmod 2^{N+1} .
$$


So part (i) is proved.

(ii) Since $v(2 l)=N+1$,

$$
(2 l+2 q+4 r \pm 1) ! ! \equiv(2 q+4 r \pm 1) ! ! \bmod 2^{N+1}
$$

by Lemma 4.2(ii). Then multiplying congruence (i) by (4.8) and noting that $(2 q+$ $4 r \pm 1) ! ! \equiv 1 \bmod 2$, the desired result follows.

(iii) To deduce part (iii), let $S=\{2,4, \ldots, 2 e\}$. Then

$$
\begin{aligned}
\prod_{x=1}^{a}(2(l+q+2 r)+x) & =\left(\prod_{i=1}^{e} 2(l+q+2 r+i)\right)\left(\prod_{x \notin S, x=1}^{a}(2(l+q+2 r)+x)\right) \\
& =2^{e}\left(\prod_{i=1}^{e}(l+q+2 r+i)\right)\left(\prod_{x \notin S, x=1}^{a}(2 l+2 q+4 r+x)\right) .
\end{aligned}
$$

Thus, using congruence (ii) $\bmod 2^{N}$ for $a=0$,

$$
\begin{aligned}
(2(l+ & q+2 r)+a) ! /\left(2^{l+q+2 r}(l+q) ! r !\right) \\
& =\left(\prod_{x=1}^{a}(2(l+q+2 r)+x)\right)(2(l+q+2 r)) ! /\left(2^{l+q+2 r}(l+q) ! r !\right) \\
& \equiv 2^{e}\left(\prod_{i=1}^{e}(q+2 r+i)\right)\left(\prod_{x \notin S, x=1}^{a}(2 q+4 r+x)\right)(2(q+2 r)) ! /\left(2^{q+2 r} q ! r !\right) \\
& \equiv(2(2+2 r)+a) ! /\left(2^{q+2 r} q ! r !\right) \bmod 2^{N+e} .
\end{aligned}
$$

(iv) To deduce part (iv), use the congruence (iii) with $e$ replaced by $e+1$ and then divide by $2^{e}$. Thus Lemma 4.5 is proved.

Lemma 4.6. Let $n=l+m, N \geq 3$ and $l=k 2^{N}, k \in \mathbf{Z}^{+}, 2 \nmid k$. Let

$$
g(a):=(-1)^{a-1}(2 a-3) ! ! /(2 a)
$$

for any integer $a>1$. Then

$$
g(n) \equiv g(m)+\left\{\begin{array}{lll}
(-1)^{(m-1) / 2} l / 2 & \bmod 2^{N+1} & \text { if } 2 \nmid m, \\
l+l /(2 m n) & \bmod 2^{N+1} & \text { if } 2 \mid m, 4 \nmid m, \\
l-l /(2 m n) & \bmod 2^{N+1} & \text { if } 4 \mid m, 8 \nmid m .
\end{array}\right.
$$

Proof. If $2 \nmid m$, then applying Lemma 4.2(ii),

$$
(2 n-3) ! !=(2 l+2 m-3) ! ! \equiv(2 m-3) ! !+2 l \bmod 2^{N+2} .
$$

By Lemma 4.1(i),

$$
(2 m-3) ! !=(2 m-3)(2(m-2)-1) ! ! \equiv(-1)^{(m-1) / 2} \bmod 4 .
$$


Since $2 \nmid m$ and $n=l+m$,

$$
1 /(m n)=1 /\left(m^{2}+m l\right) \in 1+4 \mathbf{Z}_{2}
$$

Thus

$$
\begin{aligned}
g(n)-g(m) & \equiv(2 m-3) ! !(-l /(2 m n))+l / n \\
& \equiv(-1)^{(m-1) / 2}(-l / 2)+l \\
& \equiv(-1)^{(m-1) / 2} l / 2 \quad \bmod 2^{N+1} .
\end{aligned}
$$

If $2 \mid m$, then by Lemma 4.2(i),

$$
(2 n-3) ! !=(2 l+2 m-3) ! ! \equiv-(2 l-3) ! !(2 m-3) ! ! \quad \bmod 2^{N+2+\min \{v(m), N\}} .
$$

Thus for $v(m)<N$,

$$
g(n)-g(m) \equiv\left((2 l-3) ! ! 2^{-1}(l+m)^{-1}+(2 m)^{-1}\right)(2 m-3) ! ! \bmod 2^{N+1} .
$$

If $2 \mid m$ and $4 \nmid m$, then $(2 m-3) ! ! \equiv 1 \bmod 16$ by Lemma 4.1(ii). Thus applying (4.9) and Lemma 4.2(iii) gives us

$$
\begin{aligned}
g(n)-g(m) & \equiv\left(\left(-1+2^{N+2}\right) 2^{-1}(l+m)^{-1}+(2 m)^{-1}\right)(2 m-3) ! ! \\
& \equiv 2^{N}(l / 2+m / 2)^{-1}+l(2 m(m+l))^{-1} \quad \bmod 2^{N+1}
\end{aligned}
$$

Since $l / 2+m / 2 \in 1+2 \mathbf{Z}_{2}$, we have by (4.10) that

$$
g(n)-g(m) \equiv l+l /(2 m n) \quad \bmod 2^{N+1} .
$$

If $4 \mid m$ and $8 \nmid m$, then we may assume that $m=4 \lambda$ with $2 \nmid \lambda$. Hence by Lemma 4.2(iii),

$$
(2 m-3) ! ! \equiv-1+(-1)^{(\lambda-1) / 2} 2^{4} \equiv-1+4 m \bmod 2^{6} .
$$

Also by Lemma 4.2(iii) and noting that $N \geq 3$,

$$
(2 l-3) ! !=\left(k \cdot 2^{N+1}-3\right) ! ! \equiv-1+(-1)^{(k+1) / 2} 2^{N+2} \quad \bmod 2^{N+4} .
$$

Thus using (4.9), (4.11) and (4.12),

$$
\begin{aligned}
g(n)-g(m) & \equiv\left(\left(-1+(-1)^{(k+1) / 2} 2^{N+2}\right)(2(l+m))^{-1}+(2 m)^{-1}\right)(2 m-3) ! ! \\
& \equiv\left((-1)^{(k+1) / 2} 2^{N+1}(l+m)^{-1}+l(2 m(l+m))^{-1}\right)(-1+4 m) \\
& \equiv\left((-1)^{(k-1) / 2} 2^{N+1}+2 l\right)(l+m)^{-1}-l(2 m n)^{-1} \\
& \equiv l-l(2 m n)^{-1} \bmod 2^{N+1} .
\end{aligned}
$$

The proof of Lemma 4.6 is complete. 
In the rest of this paper, we always let

$$
e:=v\left(\gamma_{u}\right)-v\left(\left(2 u_{1}\right) !\right)-\left(2 u_{3}+v\left(u_{3} !\right)\right)
$$

and define $\dot{u}$ by: $\dot{u}_{1}=\dot{u}_{3}=0$ and $\dot{u}_{i}=u_{i}$ for $i \neq 1,3$. Let $\dot{n}=w(\dot{u})$. Then $\dot{n}=n-u_{1}-$ $3 u_{3}$ and $v\left(\gamma_{\dot{u}}\right)=e$.

Lemma 4.7. Suppose that $w(u)=n$. Then $n+d-2=2\left(u_{1}+2 u_{3}+e\right)+\Gamma$, where:

(i) $\Gamma=-2$ if $\dot{n}=0$;

(ii) $\Gamma=0$ if $\dot{n}=7 \cdot 2^{\alpha}$ and $u_{7}=2^{\alpha}, \alpha \in \mathbf{N}$;

(iii) $\Gamma=1$ if $\dot{n}=2$;

(iv) $\Gamma \geq 2$ otherwise.

Proof. If $\dot{n}=0$ that is, $n=u_{1}+3 u_{3}$, we have $e=0$ and $n+d-2=2 u_{1}+4 u_{3}-2$. Then $\Gamma=-2$.

Now we assume that $\dot{n}>0$. Let $\dot{u}_{1}=\dot{u}_{3}=0$ and $\dot{u}_{i}=u_{i}(i \neq 1,3)$. Then $w(\dot{u})=$ $\dot{n}, v\left(\gamma_{\dot{u}}\right)=e$ and

$$
n+d-2=2 u_{1}+4 u_{3}+\dot{n}+\dot{d}-2 .
$$

So replacing $u$ by $\dot{u}$, in what follows we can assume that $u_{1}=u_{3}=0$. Note that $n+d=\sum_{i=1}^{n}(i+1) u_{i}$ and $e=\sum_{i=1}^{n} e_{i}$, where

$$
e_{i}:=v(i+1) u_{i}+v\left(u_{i} !\right)
$$

It follows immediately that $e_{i}>0$ if and only if either $2 \mid(i+1)$ or $u_{i} \geq 2$.

First consider case (ii): $\dot{n}=7 \cdot 2^{\alpha}$ and $u_{7}=2^{\alpha}$. Then

$$
e_{i}=3 \cdot 2^{\alpha}+v\left(2^{\alpha} !\right)=4 \cdot 2^{\alpha}-1
$$

Hence

$$
(i+1) u_{i}=8 u_{7}=2\left(e_{7}+1\right) .
$$

Since $n+d-2=8 u_{7}-2$ and $2\left(u_{1}+2 u_{3}+e\right)=2 e_{7}$, we have $\Gamma=0$.

For case (iii): $\dot{n}=2, i=2, u_{2}=1$. Clearly $e_{i}=0$. Since $u_{1}=u_{3}=e=0$,

$$
n+d-2=(i+1) u_{i}-2=1=2\left(u_{1}+2 u_{3}+e\right)+1 .
$$

Hence $\Gamma=1$.

In what follows we deal with case (iv). We claim that

$$
(i+1) u_{i} \geq 2\left(e_{i}+1\right)+2=2\left(v(i+1) u_{i}+v\left(u_{i} !\right)+1\right)+2
$$

for all cases where $i \neq 1,3$ apart from cases (ii) and (iii).

If $2 \nmid(i+1)$ and $u_{i} \geq 2$, then $e_{i}=v\left(u_{i} !\right) \leq u_{i}-1$. We can deduce that

$$
(i+1) u_{i}-2 \geq 3 u_{i}-2 \geq 2 u_{i} \geq 2\left(e_{i}+1\right) .
$$


If $2 \mid(i+1)$, we may let $i+1=\varepsilon 2^{t}$ for some $t \in \mathbf{N}$ with $2 \nmid \varepsilon$. Then $e_{i}=t u_{i}+v\left(u_{i} !\right)$, $(i+1) u_{i}-2=\varepsilon 2^{t} u_{i}-2$ and

$$
2\left(e_{i}+1\right)=2\left(t u_{i}+v\left(\left(u_{i}\right) !\right)+1\right) \leq 2(t+1) u_{i} .
$$

For $t \geq 4$, we have $2^{t}>2(t+2)$. This implies that

$$
(i+1) u_{i}-2 \geq 2^{t} u_{i}-2 \geq 2(t+1) u_{i} \geq 2\left(e_{i}+1\right) .
$$

For $t=3$, we have $i+1=2^{3} \varepsilon$. If $\varepsilon \geq 3$, then

$$
(i+1) u_{i}-2 \geq 3 \cdot 2^{3} u_{i}-2 \geq 2(3+1) u_{i} \geq 2\left(e_{i}+1\right) .
$$

If $\varepsilon=1$, then

$$
(i+1) u_{i}-2=2^{3} u_{i}-2=8 u_{i}-2
$$

and

$$
e_{i}=3 u_{i}+v\left(u_{i} !\right)=3 u_{i}+u_{i}-s\left(u_{i}\right) \text {. }
$$

Therefore

$$
(i+1) u_{i}-2 \geq 2\left(e_{i}+1\right)
$$

if $s\left(u_{i}\right) \geq 2$. But if $s\left(u_{i}\right)=1$, we have $i=7, u_{i}=2^{\alpha}$, which is case (ii).

For $t=1,2$, we have $i+1=2 \varepsilon$ or $2^{2} \varepsilon$. Since $i \neq 1,3$, we have $\varepsilon \geq 3$. Then

$$
(i+1) u_{i}-2 \geq 3 \cdot 2^{t} u_{i}-2 \geq 2(t+1) u_{i} .
$$

Hence the claim (4.13) holds as specified.

Finally, if $u_{i} \geq 1$, then $(i+1) u_{i} \geq 2$, while if $(i+1) u_{i} \geq 2\left(e_{i}+1\right)$ and $(j+1) u_{j} \geq$ $2\left(e_{j}+1\right)$, then

$$
(i+1) u_{i}+(j+1) u_{j}-2 \geq 2\left(e_{i}+e_{j}+1\right) .
$$

So we have $n+d-2 \geq 2(e+1)$ for case (iv), which follows by adding the 'local' inequalities for each value of $i$ separately.

Lemma 4.8. Let $w(u)=n$. Assume that $\dot{n}>0$. Then $v\left(\tau_{u}\right) \geq u_{3}+\lceil\dot{n} / 2\rceil-1$ except for $\dot{n}=7 u_{7}$ where $v\left(\tau_{u}\right) \geq u_{3}+\lceil\dot{n} / 2\rceil-3$.

Proof. Let $\dot{u}_{1}=\dot{u}_{3}=0$ and $\dot{u}_{i}=u_{i}(i \neq 1,3)$. Then $w(\dot{u})=\dot{n}$ and

$$
n+d-2=2 u_{1}+4 u_{3}+\dot{n}+\dot{d}-2 .
$$

So

$$
\begin{aligned}
v\left(\tau_{u}\right) & =v((n+d-2) !)-v\left(\gamma_{u}\right) \\
& =v\left(\left(2 u_{1}+4 u_{3}+\dot{n}+\dot{d}-2\right) !\right)-\left(u_{1}+v\left(u_{1} !\right)+2 u_{3}+v\left(u_{3} !\right)\right)-v\left(\gamma_{\dot{u}}\right) \\
& \geq v\left(\left(4 u_{3}\right) !\right)+v((\dot{n}+\dot{d}-2) !)-2 u_{3}-v\left(u_{3} !\right)-v\left(\gamma_{\dot{u}}\right) \\
& =u_{3}+v((\dot{n}+\dot{d}-2) !)-v\left(\gamma_{\dot{u}}\right) .
\end{aligned}
$$


Noticing that $v\left(\gamma_{\dot{u}}\right)=e=\sum e_{i}$, it suffices to prove that

$$
v((\dot{n}+\dot{d}-2) !)-\sum_{i=1}^{n} e_{i} \geq\lceil\dot{n} / 2\rceil-1 .
$$

We claim that

$$
v\left(\left((i+1) u_{i}-2\right) !\right)-e_{i} \geq\left\lceil i u_{i} / 2\right\rceil-1
$$

for $u_{i} \geq 1$ and $i \neq 1,3,7$.

If $2 \nmid(i+1)$, then

$$
e_{i}=v(i+1) u_{i}+v\left(u_{i} !\right)=v\left(u_{i} !\right)
$$

and $\left\lceil i u_{i} / 2\right\rceil=i u_{i} / 2$. Thus

$$
v\left((i+1) u_{i}-2\right) \geq v\left(u_{i} !\right)+v\left(\left(i u_{i}-2\right) !\right) \geq v\left(u_{i} !\right)+i u_{i} / 2-1 \geq e_{i}+\left\lceil i u_{i} / 2\right\rceil-1
$$

as claimed.

If $2 \mid(i+1)$, we may let $i+1=\delta 2^{t}$ where $t \in \mathbf{Z}^{+}$and $2 \nmid \delta$. Then $e_{i}=t u_{i}+v\left(u_{i} !\right)$ and

$$
(i+1) u_{i} / 2=\delta 2^{t-1} u_{i} \geq\left\lceil i u_{i} / 2\right\rceil
$$

Therefore we obtain

$$
\begin{aligned}
v\left(\left((i+1) u_{i}-2\right) !\right) & =v\left(\left(2\left(\delta 2^{t-1} u_{i}-1\right)\right) !\right) \\
& =\delta 2^{t-1} u_{i}-1+v\left(\left(\delta 2^{t-1} u_{i}-1\right) !\right) \\
& \geq\left\lceil i u_{i} / 2\right\rceil-1+v\left(\left(\delta 2^{t-1} u_{i}-1\right) !\right)
\end{aligned}
$$

Let $\delta \geq 3$. Then

$$
v\left(\left(\delta 2^{t-1} u_{i}-1\right) !\right) \geq v\left(\left(3 \cdot 2^{t-1} u_{i}-1\right) !\right) \geq v\left(\left(2 t u_{i}\right) !\right) \geq t u_{i}+v\left(\left(t u_{i}\right) !\right) \geq e_{i}
$$

since $3 \cdot 2^{t-1} u_{i}-1 \geq 2 t u_{i}$ for $t \geq 1$ and $u_{i} \geq 1$. So the claim holds for this case by (4.15).

Now let $\delta=1$, that is, $i+1=2^{t}$. Since $i \neq 1,3,7, t \geq 4$. Then by Lemma 2.1,

$$
v\left(\left(2^{t-1} u_{i}-1\right) !\right)=3 \cdot 2^{t-3} u_{i}-2+v\left(\left(2^{t-3} u_{i}-1\right) !\right) \geq t u_{i}+v\left(u_{i} !\right) \geq e_{i}
$$

since $3 \cdot 2^{t-3} u_{i}-2 \geq t u_{i}$ and $2^{t-3} u_{i}-1 \geq u_{i}$ for $t \geq 4$ and $u_{i} \geq 1$. So the claim holds for this case by (4.15).

On the other hand, if $i \neq 1,3,7$,

$$
\left.v\left(\left((i+1) u_{i}\right) !\right) \geq v\left(\left((i+1) u_{i}-2\right) !\right)\right)+1 \geq e_{i}+\left\lceil i u_{i} / 2\right\rceil
$$

by claim (4.14). If $i=7$, by Lemma 2.1,

$$
\left.v\left(8 u_{i}\right) !\right)=7 u_{i}+v\left(u_{i} !\right) \geq e_{7}+\left\lceil 7 u_{7} / 2\right\rceil .
$$


Then for $i \neq 1,3$,

$$
v\left(\left((i+1) u_{i}\right) !\right) \geq e_{i}+\left\lceil i u_{i} / 2\right\rceil .
$$

Moreover, if $u_{j} \geq 1$ for some $j \neq 7$, then by (4.14) and (4.16),

$$
\begin{aligned}
v((\dot{n}+\dot{d}-2) !) & =v\left(\left(\sum_{i=1}^{n}(i+1) u_{i}-2\right) !\right) \\
& =v\left(\left(\sum_{i=1, i \neq j}^{n}(i+1) u_{i}+(j+1) u_{j}-2\right) !\right) \\
& \geq \sum_{i=1}^{n} e_{i}+\left\lceil 2^{-1} \sum_{i=1}^{n} i u_{i}\right\rceil-1
\end{aligned}
$$

as required. Finally, if $\dot{n}=7 u_{7}$, we can compute

$$
v\left(\tau_{u}\right) \geq u_{3}+v\left(\left(8 u_{7}-2\right) !\right)-e_{7} \geq u_{3}+4 u_{7}-3-v\left(u_{7}\right) .
$$

We can easily show that

$$
4 u_{7}-v\left(u_{7}\right) \geq\left\lceil 7 u_{7} / 2\right\rceil=\lceil\dot{n} / 2\rceil .
$$

Then $v\left(\tau_{u}\right) \geq u_{3}+\lceil\dot{n} / 2\rceil-3$. Lemma 4.8 is proved.

\section{Universal Kummer congruences for powers of 2}

We are now in a position to give the universal Kummer congruences modulo powers of 2 in the form of Theorems 5.1 and 5.2. This is the second main result of this paper. We begin with Theorem 5.1.

THEOREM 5.1. Let $n \geq 2$ be an even number.

(i) If $v(n)=1$, then

$$
\begin{aligned}
\hat{B}_{n} / n \equiv- & c_{1}^{n} /(2 n)+(n / 2-1) c_{1}^{n-3} c_{3}+3(n-4) c_{1}^{n-6} c_{3}^{2} / 4 \\
& -c_{1}^{n-2} c_{2}+2 c_{1}^{n-5}\left(c_{2} c_{3}+c_{1} c_{4}\right) \bmod 4 \mathbf{Z}_{2}\left[c_{1}, \ldots, c_{n}\right]
\end{aligned}
$$

(ii) If $v(n) \geq 2$, then

$$
\begin{aligned}
\hat{B}_{n} / n \equiv( & \left.(2 n)^{-1}-2\right) c_{1}^{n}-3(n-2) c_{1}^{n-3} c_{3} / 2+(n-4) c_{1}^{n-6} c_{3}^{2} / 4+(n-8) c_{1}^{n-12} c_{3}^{4} / 4 \\
& -3 c_{1}^{n-2} c_{2}+2 c_{1}^{n-4} c_{4}+4 c_{1}^{n-4} c_{2}^{2}+(n-4) c_{1}^{n-8} c_{2} c_{3}\left(c_{3}+c_{1}^{3}\right) \\
& \bmod 8 \mathbf{Z}_{2}\left[c_{1}, \ldots, c_{n}\right] .
\end{aligned}
$$

Proof. By Lemma 4.8, and noting that $\dot{n}=\sum_{i \neq 1,3} i u_{i}$, we have only to consider the following cases.

(1) $\dot{n}=7, u_{7}=1, u_{3} \leq 1$. Then $u_{1}=n-7-3 u_{3}$. 
(2) $\dot{n}=6, u_{1}=n-6$. In this case, we have $u_{2}=3$, or $u_{2}=u_{4}=1$, or $u_{6}=1$.

(3) $\dot{n}=5, u_{5}=1$ and $u_{1}=n-5$.

(4) $\dot{n}=4, u_{1}=n-4-3 u_{3}$ and $u_{3} \leq 1$. In this case, either $u_{2}=2$ or $u_{4}=1$.

(5) $\dot{n}=2, u_{1}=n-2-3 u_{3}, u_{2}=1$ and $u_{3} \leq 2$.

(6) $\dot{n}=0, u_{3} \leq 4$. In fact, if $u_{3} \geq 5$, we have $v\left(\tau_{u}\right) \geq v\left(\left(2 u_{3}-1\right)\right.$ !) $-v\left(u_{3} !\right)-1 \geq 3$ since $\dot{n}=0$. So for our purpose, we can assume that $u_{3} \leq 4$.

For cases (1)-(3) and (4) with $u_{3}=1$, by the definition of $\tau_{u}$, we can easily check that $v\left(\tau_{u}\right) \geq 3$. We omit the details here.

For case (4) with $u_{3}=0$, if $u_{2}=2$, then

$$
v\left(\tau_{u}\right)=v((2 n-4) !)-(n-4)-v((n-4) !)-v(2 !)=1+v(n-2)=2+v(n / 2-1),
$$

that is, $\tau_{u} \equiv 0 \bmod 8$ for $v(n)=1$ and $\tau_{u} \equiv 4 \bmod 8$ for $v(n) \geq 2$. If $u_{4}=1$, then

$$
\tau_{u}=(-1)^{n-4}(2 n-5) ! /\left(2^{n-4}(n-4) ! 5\right)=2(n-3)(2 n-5) ! ! / 5 \equiv(-1)^{n / 2} 2 \bmod 8 .
$$

For case (5), if $u_{3}=2$, then

$$
v\left(\tau_{u}\right)=v((2 n-7) !)-(n-4)-v((n-8) !)-v(2 !)=v((n-4)(n-6))-1 .
$$

Hence $\tau_{u} \equiv 4 \bmod 8$ unless $n \equiv 4$ or $-2 \bmod 8$ where $\tau_{u} \equiv 0 \bmod 8$; if $u_{3}=1$, then

$$
\tau_{u}=(-1)^{n-4}(2 n-5) ! /\left(2^{n-5}(n-5) ! \cdot 3 \cdot 4\right)=(n-3)(n-4)(2 n-5) ! ! / 3 .
$$

This implies that $\tau_{u} \equiv 0 \bmod 8$ if $v(n)=2, \tau_{u} \equiv 4 \bmod 8$ if $v(n)>2$, and $\tau_{u} \equiv-n \bmod 8$ if $v(n)=1$; if $u_{3}=0$, then

$$
\tau_{u}=(-1)^{n-2}(2 n-3) ! /\left(2^{n-2}(n-2) ! \cdot 3\right)=(2 n-3) ! ! / 3 \equiv 3(-1)^{n / 2-1} \bmod 8 .
$$

For case (6), if $u_{3}=4$, then

$$
\tau_{u}=(-1)^{n-9}(2 n-10) ! /\left(2^{n-12+8}(n-12) ! 4 !\right)=-(n-5) \cdots(n-11)(2 n-11) ! ! /\left(2^{4} 3\right) .
$$

Therefore $\tau_{u} \equiv 0 \bmod 4$ if $v(n)=1$ and $\tau_{u} \equiv n / 4-2 \bmod 8$ if $v(n) \geq 2$; if $u_{3}=3$, then

$$
\begin{aligned}
v\left(\tau_{u}\right) & =v((2 n-8) !)-(n-9+6)-v((n-9) !)-v(3 !) \\
& =v((n-4)(n-6)(n-8))-2 \geq 3
\end{aligned}
$$

unless $n \equiv 2 \bmod 8$ where $v\left(\tau_{u}\right)=2$; if $u_{3}=2$, then we can derive that

$$
\tau_{u}=-(2 n-6) ! 2^{2-n}((n-6) !)^{-1} \cdot 2^{-1}=-(n-3)(n-4)(n-5)(2 n-7) ! ! / 4 .
$$

So $\tau_{u} \equiv 3(n-4) / 4 \bmod 4$ if $v(n)=1$ and $\tau_{u} \equiv(n-4) / 4 \bmod 8$ if $v(n) \geq 2$; if $u_{3}=1$, then

$$
\tau_{u}=-(2 n-4) ! 2^{1-n}((n-3) !)^{-1}=-(n-2)(2 n-5) ! ! / 2 .
$$


Thus $\tau_{u} \equiv-(n-2) / 2 \bmod 8$ if $v(n)=1$ and $\tau_{u} \equiv-3(n-2) / 2 \bmod 8$ if $v(n) \geq 2$. If $u_{3}=0$, then $\tau_{u}=-(2 n-3) ! /(2 n)$. By Lemma 4.1 , we have $\tau_{u} \equiv-1 /(2 n) \bmod 4$ if $v(n)=1$. By Lemma 4.2(iii), we deduce that if $v(n)=2$, then

$$
\tau_{u} \equiv(2 n)^{-1}\left(1+(-1)^{(n+4) / 8} 16\right) \equiv 1 /(2 n)-2 \bmod 8,
$$

and if $v(n)>2$, then

$$
\tau_{u} \equiv(2 n)^{-1}\left(1+(-1)^{\left(n 2^{-v(n)}-1\right) / 2} 2^{v(n)+2}\right) \equiv 1 /(2 n)+2 \bmod 8
$$

since $(-1)^{(a-1) / 2} \equiv a \bmod 4$ for any odd number $a$. So Theorem 5.1 is proved.

With similar methods, but in a far more complicated fashion, we have also obtained the following theorem. For the sake of brevity we omit the proof.

Theorem 5.2. Let $n=m+l, l=k 2^{N}$ with $2 \nmid k$. Let $N \geq 3$ and $m \geq 2 N+1$.

(i) If $2 \nmid m$, then

$$
\begin{aligned}
\hat{\boldsymbol{B}}_{n} / n \equiv c_{1}^{l} & \hat{B}_{m} / m+l\left(c_{1}^{n-12} c_{3}^{4}+c_{1}^{n-15} c_{3}^{5}+c_{1}^{n-5} c_{2} c_{3}+c_{1}^{n-8} c_{2} c_{3}^{2}+c_{1}^{n-7} c_{7}\right) \\
& (-1)^{(m+1) / 2} l\left(-c_{1}^{n}+c_{1}^{n-3} c_{3}+c_{1}^{n-6} c_{3}^{2}+c_{1}^{n-9} c_{3}^{3}\right) / 2 \\
& \bmod 2^{N+1} \mathbf{Z}_{2}\left[c_{1}, \ldots, c_{n}\right] .
\end{aligned}
$$

(ii) If $2 \mid m$ and $4 \nmid m$, then

$$
\begin{gathered}
\hat{B}_{n} / n \equiv c_{1}^{l} \hat{B}_{m} / m+(l+l /(2 m n)) c_{1}^{n}+l\left(c_{1}^{n-9} c_{3}^{3}+c_{1}^{n-18} c_{3}^{6}+c_{1}^{n-5} c_{2} c_{3}+c_{1}^{n-8} c_{2} c_{3}^{2}\right) \\
-l c_{1}^{n-3} c_{3} / 2+3 l c_{1}^{n-6} c_{3}^{2} / 4+\theta c_{1}^{n-12} c_{3}^{4} \bmod 2^{N+1} \mathbf{Z}_{2}\left[c_{1}, \ldots, c_{n}\right],
\end{gathered}
$$

where $\theta=-l / 2$ for $N=3$ and $\theta=l / 2$ for $N \geq 4$.

(iii) If $4 \mid m$ and $8 \nmid m$, then

$$
\begin{gathered}
\hat{B}_{n} / n \equiv c_{1}^{l} \hat{B}_{m} / m+(l-l /(2 m n)) c_{1}^{n}+l c_{1}^{n-3} c_{3} / 2+l\left(c_{1}^{n-6} c_{3}^{2}+c_{1}^{n-12} c_{3}^{4}\right) / 4 \\
+l\left(c_{1}^{n-8} c_{2} c_{3}^{2}+c_{1}^{n-5} c_{2} c_{3}\right) \bmod 2^{N+1} \mathbf{Z}_{2}\left[c_{1}, \ldots, c_{n}\right]
\end{gathered}
$$

(iv) If $8 \mid m$, then

$$
\begin{aligned}
\hat{B}_{n} / n \equiv c_{1}^{l} & \hat{B}_{m} / m-((2 n-3) ! ! /(2 n)-(2 m-3) ! ! /(2 m)) c_{1}^{n} \\
& +l c_{1}^{n-3} c_{3} / 2+5 l c_{1}^{n-12} c_{3}^{4} / 4+l c_{1}^{n-6} c_{3}^{2} / 4+l\left(c_{1}^{n-24} c_{3}^{8}+c_{1}^{n-5} c_{2} c_{3}\right. \\
& \left.+c_{1}^{n-8} c_{2} c_{3}^{2}\right) \quad \bmod 2^{N+1} \mathbf{Z}_{2}\left[c_{1}, \ldots, c_{n}\right] .
\end{aligned}
$$

\section{Acknowledgements}

The authors would like to thank Professor Shparlinski and the anonymous referee for their careful reading of the manuscript and for helpful comments and suggestions that improved its presentation. 


\section{References}

[1] A. Adelberg, 'Kummer congruences for universal Bernoulli numbers and related congruences for poly-Bernoulli numbers', Int. Math. J. 1 (2002), 53-63.

[2] A. Adelberg, 'Universal Kummer congruences mod prime powers', J. Number Theory 109 (2004), $362-378$.

[3] A. Adelberg, S. Hong and W. Ren, 'Bounds of divided universal Bernoulli numbers and universal Kummer congruences', Proc. Amer. Math. Soc. 136 (2008), 61-71.

[4] A. Baker, F. Clarke, N. Ray and L. Schwartz, 'On the Kummer congruences and the stable homotopy of BU', Trans. Amer. Math. Soc. 316 (1989), 385-432.

[5] F. Clarke, 'The universal von Staudt theorem', Trans. Amer. Math. Soc. 315 (1989), 591-603.

[6] F. Clarke and C. Jones, 'A congruence for factorials', Bull. Lond. Math. Soc. 36 (2004), 553-558.

[7] I. Dibag, 'An analogue of the von Staudt-Clausen theorem', J. Algebra 87 (1984), 332-341.

[8] J. W. L. Glaisher, 'On the residues of the sums of products of the first $p-1$ numbers, and their powers, to modulus $p^{2}$ or $p^{3}$, Quart. J. Pure Appl. Math. 31 (1900), 321-353.

[9] J. W. L. Glaisher, 'On the residues of the inverse powers of numbers in arithmetic progression', Quart. J. Pure Appl. Math. 32 (1901), 271-305.

[10] S. Hong, 'Notes on Glaisher's congruences', Chinese Ann. Math. 21B (2000), 33-38.

[11] A. Hurwitz, 'Über die Entwicklungskoeffizienten der lemniskatischen Funktionen', Math. Ann. 51 (1899), 196-226.

[12] N. M. Katz, 'The congruences of Clausen-von Staudt and Kummer for Bernoulli-Hurwitz numbers', Math. Ann. 216 (1975), 1-4.

[13] N. Koblitz, p-Adic Numbers, p-adic Analysis, and Zeta-functions, Graduate Texts in Mathematics, 58 (Springer, New York, 1984).

[14] E. E. Kummer, 'Über eine allgemeine Eigenschaft der rationalen Entwicklungscoefficienten einer bestimmten Gattung analytischer Functionen', J. reine angew. Math. 41 (1851), 368-372.

[15] E. Lehmer, 'On congruences involving Bernoulli numbers and the quotients of Fermat and Wilson', Ann. of Math. (2) 39 (1938), 350-360.

[16] H. Miller, Universal Bernoulli numbers and the $S^{1}$-transfer, Canadian Mathematical Society Conference Proceedings, 2 (American Mathematical Society, Providence, RI, 1982), 437-449.

[17] M. R. Murty, Introduction to p-adic Analytic Number Theory, Studies in Advanced Mathematics, 27 (American Math. Soc, Providence, RI, 2002).

[18] N. Ray, 'Extensions of umbral calculus I: Penumbral coalgebras and generalised Bernoulli numbers', Adv. Math. 61 (1986), 41-100.

[19] A. Robert, A Course in p-adic Analysis, Graduate Texts in Mathematics, 198 (Springer, New York, 2000).

[20] P. Tempesta, 'Formal groups, Bernoulli-type polynomials and L-series', C. R. Acad. Paris, Ser. I 345 (2007), 303-306.

[21] P. T. Young, 'Congruences for Bernoulli, Euler and Stirling numbers', J. Number Theory 78 (1999), 204-227.

[22] P. T. Young, 'Kummer congruences for values of Bernoulli and Euler polynomials', Acta Arith. 99 (2001), 277-288.

[23] J. Zhao, 'Wolstenholme type theorem for multiple harmonic sums', Int. J. Number Theory 4 (2008), 73-106.

\section{SHAOFANG HONG, Yangtze Center of Mathematics, Sichuan University, Chengdu 610064, PR China}

and

Mathematical College, Sichuan University, Chengdu 610064, PR China

e-mail: sfhong@scu.edu.cn, s-f.hong@tom.com, hongsf02@yahoo.com 
JIANRONG ZHAO, School of Economic Mathematics, Southwestern University of Finance and Economics,

Chengdu 610074, PR China

e-mail: mathzjr@foxmail.com

WEI ZHAO, Science and Technology on Communication Security Laboratory, Chengdu 610041, PR China

e-mail: zhaowei9801@163.com 